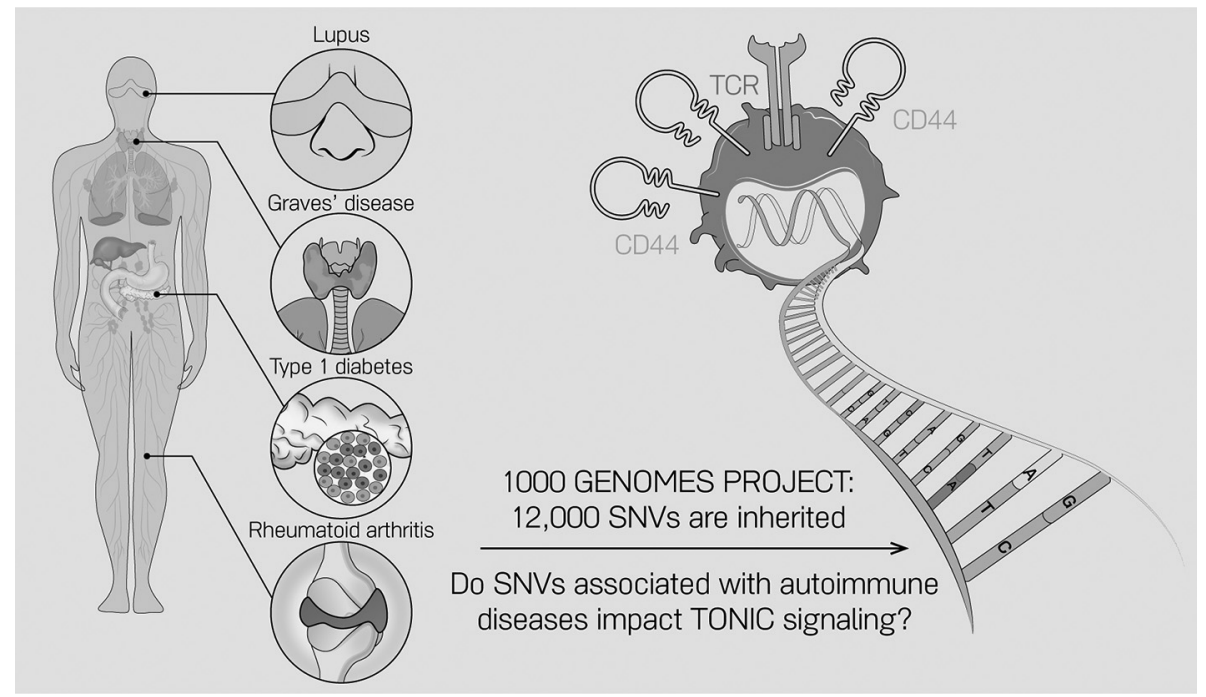

Abstract 196 Figure 1 SNVs and Tonic Signals in T cells

constitutes an unexplained maintenance cue. Our studies of a mouse model with a single nucleotide variant in Rasgrp1, Rasgrp1Anaef, and autoimmune features suggested that basal mTOR signals impact the resting state of $\mathrm{T}$ cells in vivo (Daley et al., 2013). We set out to investigate if tonic signals in primary $\mathrm{T}$ cells establish a primed yet controlled state.

Methods A sophisticated mouse model allowing for inducible deletion of the adapter LAT in T cells was coupled to quantitative biochemical analyses of primary $\mathrm{T}$ cells, gene expression analyses, and functional $\mathrm{T}$ cell assays, Through our Rasgrp1Anaef mouse model, quantitative biochemical analyses of primary $\mathrm{T}$ cells, and genome-wide ribosome profiling, we established that tonic mTORC1 signals shape the baseline translational landscape in resting $\mathrm{T}$ cells in vivo.

Results In 2017, we described how tonic signals prevent aberrant basal activity of naïve T cells; Tonic signals through LAT (Linker for activation of $\mathrm{T}$ cells) and the transcriptional regulator HDAC7 maintain mRNA expression of a cluster of target genes that are negative regulators of spurious $\mathrm{T}$ cell proliferation and Th2 differentiation (Myers et al., 2017a).

We recently discovered robust and selective mTORC1 kinase signals in resting, naive $\mathrm{CD} 4+\mathrm{T}$ cells. We find that RasGRP1 is necessary and sufficient to generate tonic mTORC1 signals. These tonic mTORC1 signals govern a baseline translational program and impact the capacity of CD4 + T cells to take on effector functions (Myers et al., in revision). Aberrantly increased tonic mTORC1 signals in a Rasgrp1Anaef mouse model (Daley et al., 2013) drive immune pathology, alter the ribosome profiles of resting $\mathrm{T}$ cells, and enhance differentiation to the Tph, Tfh, and Th2 fates.

Conclusions Our fundamental studies on tonic signals demonstrate a dynamic balance in naive $\mathrm{T}$ cells with an inhibitory arm regulated through tonic gene expression and a stimulatory arm by shaping a basal translational landscape that includes a mTOR signature. Dysregulation of mTORC1 signaling has been implicated in autoimmune diseases and $\mathrm{T}$ cells from systemic lupus erythematosus (SLE) patients exhibit mTORC1 activation (Perl, 2016). Single nucleotide- and splice- variants of Rasgrp1 have been implicated in SLE (Yasuda et al., 2007), and Type 1 Diabetes and Graves disease (Plagnol et al., 2011; Qu et al., 2009). The mTORC1 inhibitor rapamycin can block $\mathrm{T}$ cell activation in SLE patients and has therapeutic efficacy in SLE (Perl, 2016). We believe that our fundamental studies provide a novel platform for investigating tonic signal activity in $\mathrm{T}$ cells from autoimmune patients and will discuss latest progress.

Funding Source(s): NSF-GRFP (1650113 to DRM) and the NIH-NIAID (R01-AI104789 and P01-AI091580 to JPR).

Are altered tonic signals in $\mathrm{T}$ cells a common consequence of single nucleotide variants (SNVs)?

\section{RACIAL DISPARITIES IN LUPUS MEDICATION ADHERENCE}

Kai Sun*, Amanda M Eudy, Jennifer L Rogers, Lisa G Criscione-Schreiber, Jayanth Doss, Rebecca E Sadun, Megan EB Clowse. Duke University

\subsection{6/lupus-2019-Ism.197}

Background Medication non-adherence is common among systemic lupus erythematosus (SLE) patients and is disproportionately higher among minorities. We aimed to examine the adherence gap between minority and Caucasian SLE patients and explore factors associated with non-adherence that may present avenues for intervention.

Methods Cross-sectional data were obtained via survey and chart review from consecutive SLE patients with 1 scheduled lupus medication. The Medication Adherence Self-Report Inventory (MASRI) was used to estimate adherence in the preceding month from 0\%-100\%. High Self-reported Adherence was defined as MASRI 90\%. Pharmacy refill data in the preceding 3 months were obtained by phone calls. High Refills was defined as a medication possession ratio of $80 \%$ for all prescribed SLE medications. Additional covariates included: positive and negative affect, self-efficacy, patient-reported health status, patient-rated interactions with providers, rheumatic medication regimen complexity, SLE Disease Activity Index (SLEDAI), and physician global assessment for inflammatory (PGA1) and noninflammatory (PGA2) symptoms. Adherence groups were compared.

Results 84 enrolled (37\% Caucasians, 59\% African American, $1 \%$ Native American, and 4\% Hispanic). Median age was 42 (range 22-68), 95\% were female, 49\% had college education, and $49 \%$ had private insurance. 54 (64\%) had High Self- 
reported Adherence, but only 42 (50\%) had High Refills. Minorities had lower rates compared to Caucasians for both High Self-reported Adherence $(55 \%$ vs $81 \%, p=0.02)$ and High Refills ( $40 \%$ vs. $68 \%, \mathrm{p}=0.01)$. Among 52 taking disease modifying agents (DMARDs) including methotrexate, leflunomide, azathioprine, and mycophenolate, High Refill rate was only $46 \%$, and the difference between minorities and Caucasians was even larger $(35 \%$ vs. $73 \%, p=0.01)$. In this group, the Low Refill group had higher SLEDAI (4 vs 2, $\mathrm{p}=0.02$ ), PGA2 ( 1 vs $0, \mathrm{p}=0.04$ ), and a trend for living with children $(41 \%$ vs. $17 \%, \mathrm{p}=0.07)$ compared to those with High Refill rates. There were no statistically significant differences between the DMARD High and Low Refill groups in terms of positive and negative affect, self-efficacy, patient-provider interactions, patient self-reported outcomes, and other clinical factors.

Conclusions Significant racial disparities exist in lupus medication adherence, and the gap is even more substantial for patients taking DMARDs, medications crucial for the treatment of more severe lupus manifestations. This gap likely contributes to the known racial disparities in SLE outcomes. We did not discover potential modifiable factors in this small sample. Qualitative studies are needed to better understand this racial gap and to develop interventions that address racial disparities in SLE.

Funding Source(s): 5U54MD012530-02

\section{IFN-KINOID IN SYSTEMIC LUPUS ERYTHEMATOSUS (SLE): RESULTS FROM A PHASE 2B, RANDOMIZED, PLACEBO-CONTROLLED STUDY}

${ }^{1}$ Frédéric Houssiau*, ${ }^{2}$ Aikaterini Thanou, ${ }^{3}$ Minodora Mazur, ${ }^{4}$ Edgar Ramiterre, ${ }^{5}$ Danny Alexis Gomez Mora, ${ }^{6}$ Maria Misterska-skora, ${ }^{7}$ Risto Perich Campos, ${ }^{8}$ Svetlana Anatolyevna Smakotina, ${ }^{9}$ Sergio Cerpa Cruz, ${ }^{10}$ Bassem Louzir, ${ }^{11}$ Therese Camille, ${ }^{12}$ Michael Tee. ${ }^{1}$ Service de Rhumatologie, Cliniques universitaires Saint-Luc, Brussels and Pôle de Pathologies Rhumatismales Inflammatoires et Systémiques, Université catholique de Louvain; ${ }^{2}$ Research Affiliate Arthritis and Clinical Immunology Research Program Oklahoma Medical Research Foundation; ${ }^{3}$ University Professor at State Medical and Pharmaceutical University; ${ }^{4}$ Section of Rheumatology, Department of Internal Medicine, Southern Philippines Medical Center JP Laurel Ave, Davao City Philippines; ${ }^{5}$ Rheumatologist from UNIVERSIDAD NACIONAL DE COLOMBIA Healthy Medical Center Kra 4 \# 7-39 Zipaquira; ${ }^{6}$ Medical Center Oporów Solskiego 4a/1 52-416 Wrocaw; ' Rheumatology Department, Hospital Nacional Guillermo Almenara Irigoyen. EsSalud. Lima- Perú. Universidad Nacional Mayor de San Marcos; ${ }^{8}$ Doctor of medical sciences, professor of Kemerovo State Medical University; ${ }^{9}$ Attending Physician and Associated Clinical Professor Medicine/Rheumatology Department Hospital Civil de Guadalajara; ${ }^{10}$ Head of Department of Internal Medicine Military hospital of Tunis 1008 Tunis; ${ }^{11}$ Neovacs; ${ }^{12}$ Vice Chancellor Medical Center Manila, Rheumatology Department UP Manila College of Medicine and ManilaMed

\subsection{6/lupus-2019-Ism.198}

Background The immunotherapeutic vaccine Interferon-kinoid (IFN-K) consists of a heterocomplex of inactivated recombinant human IFN-2b coupled to a T-helper carrier protein, Keyhole Limpet Haemocyanin. A phase I/IIa was published. Here, we report the results of a 36 week (w) phase $2 b$, randomized, double-blind, placebo-controlled (PBO), multi-center study assessing the efficacy and safety of IFN-K in patients with active SLE on standard of care therapy.

\begin{tabular}{llll} 
Abstract 198 Table 1 & & \\
\hline & IFN-K & Placebo & p value \\
\hline IFN gene signature reduction & $-31.3 \%$ & $-0.4 \%$ & $<0.0001$ \\
Modified BICLA & $35(41.2 \%)$ & $29(34.5 \%)$ & 0.33 \\
SRI(4) & $57(67.9 \%)$ & $54(65.1 \%)$ & 0.62 \\
SRI(4) with CS $\leq 5 \mathrm{mg} / \mathrm{d}$ & $43(54.4 \%)$ & $30(39 \%)$ & 0.07 \\
SRI(4) with CS $\leq 5 \mathrm{mg} / \mathrm{d}$ & $40(55.6 \%)$ & $30(39.0 \%)$ & 0.04 \\
(IFN-K subgroup with neutralizing Abs) & & & \\
SRI(4) with CS $\leq 7.5 \mathrm{mg} / \mathrm{d}$ & $46(58.2 \%)$ & $33(42.9 \%)$ & 0.07 \\
SRI(4) with CS $\leq 7.5 \mathrm{mg} / \mathrm{d}$ & $43(59.7 \%)$ & $33(42.9 \%)$ & 0.04 \\
(IFN-K subgroup with neutralizing Abs) & & & \\
LLDAS & $45(52.9 \%)$ & $25(29.8 \%)$ & 0.002 \\
Mean CS dose * & $5.4 \mathrm{mg} / \mathrm{d}$ & $7.1 \mathrm{mg} / \mathrm{d}$ & 0.009 \\
\hline
\end{tabular}

*The mean daily CS dose was lower in the IFN-K group from w28 onwards.

Methods SLE patients (4 ACR criteria) with moderate to severe disease activity (SLEDAI 2K 6 and 1 BILAG A and/or 2 BILAG B scores); positive IFN gene signature; and ANA and/or anti-dsDNA, were randomized (1:1) to 5 IM injections of IFN-K or PBO at days $0,7,28$, and months 3 and 6. Coprimary objectives at $\mathrm{w} 36$ were neutralization of IFN gene signature and BICLA response modified by mandatory corticosteroid (CS) tapering ( $5 \mathrm{mg} / \mathrm{d}$ prednisolone equivalent) by w24 with no increase to w36. Secondary objectives at w36 were SRI(4) and SRI(4) with CS tapering (5 mg or $7.5 \mathrm{mg} / \mathrm{d}$ prednisolone equivalent) by w36, Lupus Low Disease Activity State (LLDAS), safety and immunogenicity.

Results Among 185 patients randomized, 91 and 93 were respectively treated with IFN-K and $\mathrm{PBO}$, and 85 (92.4\%) and $84(90.3 \%)$ completed the study. Seventy-two of 79 (91.1\%) IFN-K treated patients (Per Protocol Set) developed anti-IFN neutralizing antibodies (Abs). Primary and secondary outcome measures at w36 are detailed in the Table:

IFN-K was well tolerated, with similar rates of treatmentemergent adverse events (TEAEs $82.4 \%$ vs $76.3 \%$ ) and TEAEs leading to study drug discontinuation $(4.4 \%$ vs. $4.3 \%)$ in the IFN-K and PBO groups, respectively. Serious adverse events (SAEs) were more common on $\mathrm{PBO}$ vs IFN-K $(12.9 \%$ vs $6.6 \%)$. Cancer $(n=4)$ and lupus nephritis $(n=2)$ were reported in the PBO group and there was one severe infection in the IFN-K group. One death occurred in each group.

Conclusions IFN-K induced neutralizing anti-IFN Abs in $91.1 \%$ of treated patients and significantly reduced IFN gene signature. Modified BICLA at w36 did not differ between IFN-K and PBO. Trends on SRI (4) with steroid tapering at w36 favored IFN-K, and became significant when patients exhibiting neutralizing Abs were included in the exploratory analysis. Furthermore achieving a Lupus Low Disease Activity State discriminated the two groups at w36, in favor of IFN-K. A significant CS sparing effect of IFN-K was observed from w28 onwards. The safety profile of IFN-K was acceptable. Results merit further evaluation in phase 3 studies.

Funding Source(s): The study was funded by Neovacs. 